\title{
Interactions between polygenic scores and environments: Methodological and conceptual challenges
}

\author{
Benjamin W. Domingue 1* \\ Sam Trejo 1 \\ Emma Armstrong-Carter 1 \\ Elliot M. Tucker-Drob 2
}

1. Stanford University

2. University of Texas at Austin

* bdomingue@stanford.edu

Acknowledgements: This work has been supported by the Russell Sage Foundation and the Ford Foundation (Grant No. 96-17-04). ST was supported by the National Science Foundation (Grant No. DGE-1656518) and the Institute of Education Sciences (Grant No. R305B140009). EMTD was supported by the National Institutes of Health (Grant No. R01AG054628 and R01HD083613) and by the Jacobs Foundation. Any opinions expressed are those of the authors alone and should not be construed as representing the opinions of any foundation. 


\begin{abstract}
Interest in the study of gene-environment interaction has recently grown due to the sudden availability of molecular genetic data — in particular, polygenic scores — in many long-running cohort studies. Identifying and estimating statistical interactions comes with several analytic and inferential challenges; these challenges are heightened when used to integrate observational genomic and social science data. We articulate some of these key challenges, provide new perspectives on the study of gene-environment interactions, and finally offer some practical guidance for conducting research in this area. Given the sudden availability of well-powered polygenic scores, we anticipate a substantial increase in research testing for interaction between such scores and environments. The issues we discuss, if not properly addressed, may impact the enduring scientific value of gene-environment interaction studies.
\end{abstract}




\section{Introduction}

Over the past decade, the world has witnessed a massive expansion of our ability to conduct biological inquiry into human behavior. As a result of the plummeting cost of DNA sequencing, combined with computational developments in the processing of genetic data, researchers have made great strides linking genetic variation to human behavior (1). The genome-wide association study (GWAS) has emerged as a key tool in this endeavor $(1,2)$. Among the most influential discoveries from the first decade of GWAS is that a broad array of both behavioral traits (e.g. mental wellbeing, cognitive function, and risk-taking) and biomedical traits (e.g. height, body-mass index, and cardiovascular disease) are highly polygenic. In other words, the genetic bases of population variation in these traits is attributable to many genetic variants each with a small effect (rather than a few variants with large effects) $(3,4)$.

The prevalence of polygenic architectures has led many researchers interested in engaging with recent genetic findings to forego the study of specific genetic variants in favor of genome-wide composite measures (5). These composite measures, known as polygenic scores (PGSs), summarize the cumulative effects of many variants across the genome and aim to index an individual's genetic liability for a given trait. PGSs aggregate effect size estimates for millions of genetic variants. Although these effect size estimates, or allelic weights, are individually tiny, their combined effect is robustly predictive of a sizable proportions of variance in consequential outcomes $(5,6)$, such as educational attainment and lifespan (7-9). ${ }^{1}$ The use of PGSs has spurred enthusiasm for genetics research to advance our understanding of the behavioral and biomedical sciences (10-13).

However, this increased focus on genetic influences does not diminish interest in the environment. Indeed, how social and environmental factors combine and interact with biological factors to produce individual differences is a question at the forefront of many research agendas in the behavioral sciences. Researchers have long posited that genetic effects likely vary as a function of environment (14). ${ }^{2}$ For example, in the twin study literature, there has been substantial interest in whether decompositions of observed variation in a phenotype into genetic

\footnotetext{
${ }^{1}$ Exactly how predictive a PGS is of a given trait depends on both on the trait's heritability and the sample size of the GWAS used to derive the effect size estimates, see Figure 2 of (10).

${ }^{2}$ We note that one could alternatively discuss environmental effects differing as a function of genetics; we utilize the original formulation in this paper but note that the latter may occasionally be the more germane.
} 
and environmental components differ by socioeconomic context or age (15). Other research designs have tested interactions between specific genetic variants and environmental features. In spite of the intuitive appeal of such an approach, it has proven technically challenging to implement (16). Indeed, a first wave of such candidate $\mathrm{GxE}$ research has become infamous for widespread replication failures (17). Such failures may partly be attributable to unrealistically large expectations for effect sizes of individual variants. Although we may potentially circumvent this issue by compiling many individual small effects to produce a PGS, other challenges remain. Further, as we discuss, even when GxE results are robust and replicable, the interpretational and practical implications of such research can be unclear.

The increasing availability of PGSs — datasets such as the Health and Retirement Study (18), Add Health (19), and the Wisconsin Longitudinal Study (20) are posting pre-constructed scores for use by researchers and catalogues of polygenic scores are being made available (21) offers new, potentially more robust, avenues for exploration of GxE. Given the emergence of this new tool, we aim to provide timely guidance on how to conduct high-quality GxE research using PGSs. In this paper, we have two main objectives. First, we outline several problems associated with performing GxE research. Some of these problems (i.e., endogeneity and specification error) are generic problems that are also relevant to the specific context of GxE; others, which we discuss in more detail, involve considerations specifically applicable to the study of GxE. Second, we offer some guidelines for designing, implementing, and interpreting high-quality GxE research using PGSs.

\section{The standard GxE model}

We consider some outcome, Y, to be a function of an individual's genotype, G, and some (potentially continuously varying) environmental exposure, E. We generically describe this datagenerating model as

$$
\mathrm{E}(\mathrm{Y} \mid \ldots)=\mathrm{f}(\mathrm{G}, \mathrm{E}) .(\text { Eqn } 1)
$$

Eqn 1 accommodates both complex interplay between genotype and environment as well as outcomes that are not normally distributed (e.g., Y may have a Bernoulli distribution). We 
supplement this simple model with a few crucial assumptions. We assume that we have reasonable proxies available for $\mathrm{G}$ and $\mathrm{E}$ and some identifiable approximation to $\mathrm{f}()$. We comment on each of these assumptions below.

With respect to $G$, we assume that we can characterize genetic influence on the trait as a PGS,

$$
\mathrm{PGS}_{\mathrm{i}}=\Sigma \beta_{\mathrm{j}}(\mathrm{N} \text { Alleles })_{\mathrm{ij}}
$$

i.e., a sum wherein the number $(\mathrm{N})$ of alleles (typically 0,1 , or 2 ) that an individual $i$ has for each SNP $\mathrm{j}$ is weighted by the effect, $\beta_{\mathrm{j}}$, identified via GWAS. We note a few assumptions implicit in the above. We are focusing on traits that have a genetic architecture appropriately characterized by effects that are additive with respect to one another (but may still be nonadditive in terms of their potential to interact with environmental contexts) and dispersed over many loci. We view the assumption of additivity as an acceptable simplification given both the success of additive GWAS and the relative lack of strong empirical support for dominance or epistasis (i.e., GxG) models (22).

The assumption that genetic effects spread over many loci is not especially restrictive. Empirical work has indicated that many traits of interest in population health-BMI, cardiovascular disease — are highly polygenic. Not all traits_consider monogenic diseases such as cystic fibrosis - necessarily have this characteristic. However, as the sample size of the GWAS used to generate a PGS increases, weights (i.e., $\beta_{\mathrm{j}}$ ) for SNPs that are not relevant to the phenotype of interest will go towards zero and thus a polygenic approach can still potentially be used in such cases. Moreover, much of our discussion still applies when using genetic predictors constructed from a smaller number of variants or even using a single variant allele count $(23,24)$.

We also note that GWAS results (i.e., $\beta_{\mathrm{j}}$ ) are themselves potentially a function of both trait-specific biology and contextual features of the data used to derive them (e.g., the social and policy landscape governing behavior of participants in the GWAS, selection issues associated with being a part of GWAS sample, etc.) $(25,26)$. Therefore, PGSs index the genetic propensity within the environmental context and demographic characteristics of participants in the original 
discovery GWAS on which the PGS is constructed. ${ }^{3}$ An interaction between PGS and environment may then indicate that the role of genetic factors on the outcome is higher in some environments than others, that the sample in one environment is more similar to the sample from the discovery GWAS than in others, or some mixture of the two. This ambiguity regarding interpretation should be kept front of mind when findings from polygenic score research are interpreted. However, we focus the current article on inferential and statistical issues primarily pertaining to the samples in which the PGSs are constructed and analyzed.

With respect to E, we assume that researchers use specific measures of the environment, which we denote ENV. At present, research typically focuses on variation in measured environments that have relatively large main effects on Y. We consider this topic in detail below. In general, we emphasize that there are numerous challenges associated with identification of the appropriate ENV in GxE research (28). Are we measuring the environmental characteristic at the appropriate level (e.g., household versus neighborhood versus community)? Are we measuring a salient exposure given the respondents ages? Can we measure the environmental exposure of interest with high fidelity? Are the exposures and contexts of interest correlated with other, unmeasured environmental or genetic variables that are themselves the driving forces in the identified GxE? The identification of appropriate ENV measures may merit additional scrutiny (see also the suggestion in Section 4 related to looking for variation using more agnostic methods).

Finally, we assume that the unknown function $\mathrm{f}()$ is well-approximated by a relatively simple model. In particular, many GxE studies aim to shed light on Eqn 1 using regression models of the form

$$
\mathrm{E}(\mathrm{Y} \mid \ldots)=\mathrm{b} 0+\mathrm{b} 1 \cdot \mathrm{PGS}+\mathrm{b} 2 \cdot \mathrm{ENV}+\mathrm{b} 3 \cdot \mathrm{PGS} \cdot \mathrm{ENV}+\text { covariates. }(\text { Eqn } 2)
$$

The aim is to have Eqn 2 elucidate key properties of the (unknown) data generating process, even if Eqn 2 is only a rough approximation of Eqn 1. There are several concerns that apply to such regression models that have been discussed in the context of GxE research. We review two important issues that have been the subject of previous scrutiny in Sections 2A and 2B below.

\footnotetext{
${ }^{3}$ In practice, polygenic scores may contain information on correlated non-genetic factors (for example, population stratification and dynastic effects like genetic nurture) in addition to true genetic propensity (27).
} 
We then consider several novel issues of specific relevance when conducting GxE studies in Section 3.

\section{A. Endogenous environments}

Environmental exposures are typically partly endogenous (29), creating complex patterns of correlations between genes, focal environments, and other relevant exposures that lead to inferential challenges for the identification of GxE (30-32). Because environments are typically not randomly assigned - though there are a few exceptions (33-35) - both genetic and nongenetic factors play a role in determining an individual's likelihood of encountering a particular environmental exposure. This induces a correlation between an individual's actual exposure to a given environment and all the genetic and non-genetic factors that correlate with their likelihood of exposure to that environment. Thus, estimation of Eqn 2 may yield a significant value of the interaction parameter (b3) even when this parameter is in fact zero in the true model. This spurious detection of $\mathrm{GxE}$ is due to omitted variable bias resulting from a failure to control for the full suite of genetic and non-genetic factors that select people into environments.

Consider the example of a researcher interested in testing whether GxE exists between genes for body-mass index, as characterized by a body-mass index PGS (36), and municipal soda taxes. Both genetics and non-genetic individual characteristics partially determine an individual's likelihood of residing in a municipality with soda taxes. If a researcher fits Eqn 2 and finds a statistically significant $b 3$, such a finding could have a number of explanations. It might represent (A) detection of true GxE between the body-mass index PGS and soda taxes. However, it could also represent (B) detection of GxE between the BMI PGS and some other city-level environment correlated with soda taxes (for example, the presence of a robust social welfare system). It could also be due to other forms of non-GxE interaction: (C) detection of GxG between the body-mass index PGS and the regions of the genome that are disproportionally over- or under-represented in people living in cities with soda taxes or (D) detection of GxP between the body-mass index PGS and another phenotype, P, that tends to be disproportionally over- or under-represented in people living in cities with soda taxes. For that matter, it could be due to some combination of A-D. The endogeneity of environmental exposures seriously complicates interpretation of $b 3$ and in turn the identification of genuine GxE. Approaches to 
estimating GxE using exogenous environments are possible (33-35) but obviously hinge on the existence of experimental or quasi-experimental variation in a given dataset.

\section{B. Model misspecification}

Misspecification bias is a generic problem that introduces additional complexities in the case of interaction research. For example, care must be taken to distinguish between models containing interactions between two variables versus those with no interactions but nonlinear (e.g. quadratic) terms in one or both of the two variables $(37,38)$. It can be challenging to adjudicate between these alternatives, yet they offer distinct perspectives on the underlying data generating process.

GxE research must also attend to the issue raised by Keller (39) focusing on the covariates included in Eqn 2. When covariates are included in Eqn 2, specification error may result if additional interaction terms between the covariates and both E and the PGS are not included. This is because the main effects of the covariates are insufficient controls in the case where there is covariation between both the covariate and genotype or the covariate and the environment. Unlike the case of endogenous environments, there is a straightforward solution; researchers simply need include a full suite of interaction terms between the PGSs and the covariates when estimating Eqn 2.

\section{Study design issues in GxE research}

\section{A. The environmental exposure}

A great deal of research in the social sciences focuses exclusively on the effects of environments. For example, there is substantial interest in the effects of poverty, reflected primarily in the home environment of a young child, on the developing brain and related cognitive functioning $(40,41)$. GxE research has tended to emphasize environmental variables like poverty for which large main effects have been well documented (42-44). However, the environmental features having large main effects need not also be the features that lead to nuanced GxE effects. GxE research may benefit from additional attention to the theorized nature of the candidate environmental variables deployed in GxE research. 
To better frame our argument, we consider two stylized patterns of GxE interaction. We emphasize here that these two patterns are not meant to act as an exhaustive taxonomy of GxE interaction. Rather, they serve as illustrations of the sorts of considerations that we encourage. First, consider GxE interactions in which the environmental functions as a "dimmer" on genetic effects. Dimmers, as in switches responsible for dimming or brightening lights, may magnify or constrict genetic effects on an outcome without changing their sign. Investigating dimmer-type GxE may be of high substantive interest in many contexts. For instance, it is of strong practical and theoretical importance to determine whether an educational policy with a robust positive average effect for the population disproportionally benefits children at highest genetic risk, those at lowest genetic risk, or has uniform effect across the spectrum of genotypes.

However, as we discuss at greater length in the following Section 3B, it is also important to be vigilant about the potential for $\mathrm{GxE}$ to arise as an artifact of more general effects on the distribution of the observed outcome itself. For instance, suppose the educational policy of interest is associated with an appreciable increase in both the mean and variation of math achievement in the student population. It is then possible that the intervention has increased the effect of the PGS on math achievement (i.e., a positive b3 estimate in Eqn 2) simply as a byproduct of more general increases in variation in math achievement. Because conventional ordinary least squares methods are blind to this type of heteroscedasticity, the concomitant increase in non-PGS variance may go overlooked.

Second, consider GxE interactions in which the environment functions as a lens on genetic effects. An environment acts as a lens when the direction of the effect of the PGS differs across the range of that environment. We refer to these environments as lenses based on the optical notion of a lens; in particular, certain glass lenses invert the orientation of objects. When considering lenses, the relative effect of a given genotype may be positive for a "low" level of the relevant environmental exposure and negative for "high" levels of the exposure, or vice versa. This has led to the hypothesis that what qualifies as a high or low risk genotype may depend upon the environmental context (45-47). Note that an environment may function as a lens even when it has a limited main effect.

Environments conceptualized to operate as lenses are typically used as a theoretical motivation for doing GxE research typically and yet, in practice, many of the environmental measures typically used in GxE studies may be conceptually closer to the dimmer category. 
Moreover, the selection of PGS effects for examining lens-type GxE may be particularly challenging in that we construct PGSs from GWASs that only include main effects of SNPs. If the environmental context of the participants in the GWAS sample used to construct the PGS is similar to that in the test sample used to estimate GxE, then it is unlikely to include SNPs that demonstrate lens-type patterns as the main effects of these SNPs will be close to zero.

We can also understand the difference between a dimmer and a lens in terms of their effect on the rank ordering of outcomes. All else being equal, a dimmer is order-preserving; that is, it preserves the order of the genotypes at different levels of the environment. Variation in the dimmer serves to vary the distance, in the outcome metric, between different levels of the PGS but never changes the rank-orderings of the levels. In contrast, a lens reverses the order of genotypes; a PGS that predicts an outcome near the top of the distribution at one level of environmental exposure will predict an outcome near the bottom at another level of environmental exposure. Our dimmer/lens typology is similar in many respects to the ordinal/disordinal typology previously suggested (48) but may be a more useful conceptual distinction as GxE research becomes more common in the social sciences.

\section{B. Coarsened outcome variables}

Characteristics of the distribution of Y may have crucial implications for conducting GxE studies. When $\mathrm{Y}$ is a discrete outcome coarsened from an underlying continuous variable, researchers encounter an opportunity to misinterpret affirmative findings of GxE. For simplicity of exposition, we focus on the simplest case where $\mathrm{Y}$ is dichotomous (though the phenomenon extends to coarsened variables that take more than two values).

Suppose a dichotomous outcome $\mathrm{Y}$ is a coarsened version of some continuously varying latent indicator $\mathrm{Y}^{*}$ (so, $\mathrm{Y}=1$ if $\mathrm{Y}^{*}>\lambda$ for some scalar $\lambda$ and 0 otherwise). For example, $\mathrm{Y}$ might be obesity or college completion (in which case $\mathrm{Y}^{*}$ would be body mass index or years of schooling, respectively). Suppose we estimate Eqn 2 with OLS using Y* instead of Y and yield a non-zero and statistically significant b3. How should we interpret such a finding? One possibility is that a finding of GxE suggests changes in the slope of association between $G$ and $Y^{*}$. This, we argue, is what researchers generally have in mind when conducting studies testing for GxE. However, a second possibility is that a purely environmental shock may shift the intercept of the 
association line between $\mathrm{G}$ and $\mathrm{Y}^{*}$ thus resulting in a $\mathrm{GxE}$ finding (i.e. a non-zero and statistically significant b3).

We illustrate the basic problem in Figure 1. When we examine relationships between PGS and outcome in the context of the continuously measured version ( $\mathrm{Y}^{*}$ in Panel A), we observe a constant linear association with genotype across two environments. However, when we observe a dichotomized version of the outcome ( $\mathrm{Y}$ in Panel B), we have a relationship that is more challenging to interpret. In particular, Panel B suggests GxE when a linear probability model is used (i.e. the dotted curves are not parallel). In contrast, when a logistic regression model is used, estimates of GxE (i.e. B3) are not biased but may suffer from low power (and large confidence intervals) due to low variability in the dichotomized outcome at some regions of the environmental measure. This problem may be even more severe when gene-environment correlation results in a large shift in the distributions of the PGS along the range of the environmental measure.

Findings such as those in Figure 1 Panel B are worth noting and they may be highly relevant in cases where the continuous $\mathrm{Y}^{*}$ is of less interest than the dichotomized $\mathrm{Y}$ (for example, college completion may well matter more than years of schooling) or when $\mathrm{Y}^{*}$ is latent. However, we also need not confuse matters by misunderstanding the nature of the associations in question. If findings are driven by changes in intercepts and relatively consistent slopes (i.e., Panel A), researchers should say as much. In general, attention should be paid to distinguishing between these two possibilities; see also our discussion of this issue in an empirical context elsewhere (49). ${ }^{4}$

Figure 1. The challenge of studying GxE with dichotomous outcomes. Panel A: True association between PGS and continuously varying outcome $\mathrm{Y}^{*}$. Densities show distributions above horizontal blue line for those in high and low environments. Panel B: True associations and those estimated using either a logistic regression model or a linear probability model when $\mathrm{Y}^{*}$ has been dichotomized prior to analysis $\left(\mathrm{Y}=1\right.$ when $\left.\mathrm{Y}^{*}>\lambda\right)$. The linear probability model (lpm) produces the misimpression of GxE (non-parallel regression lines). The logistic regression model

\footnotetext{
${ }^{4}$ See also a similar observation in a different context: https://twitter.com/Joni_Coleman/status/1220332653599186946?s=20
} 
does not suffer from this bias, but may still suffer from large standard errors and low power when we observe low variability in the dichotomous $\mathrm{Y}$ variable in one of the environments.
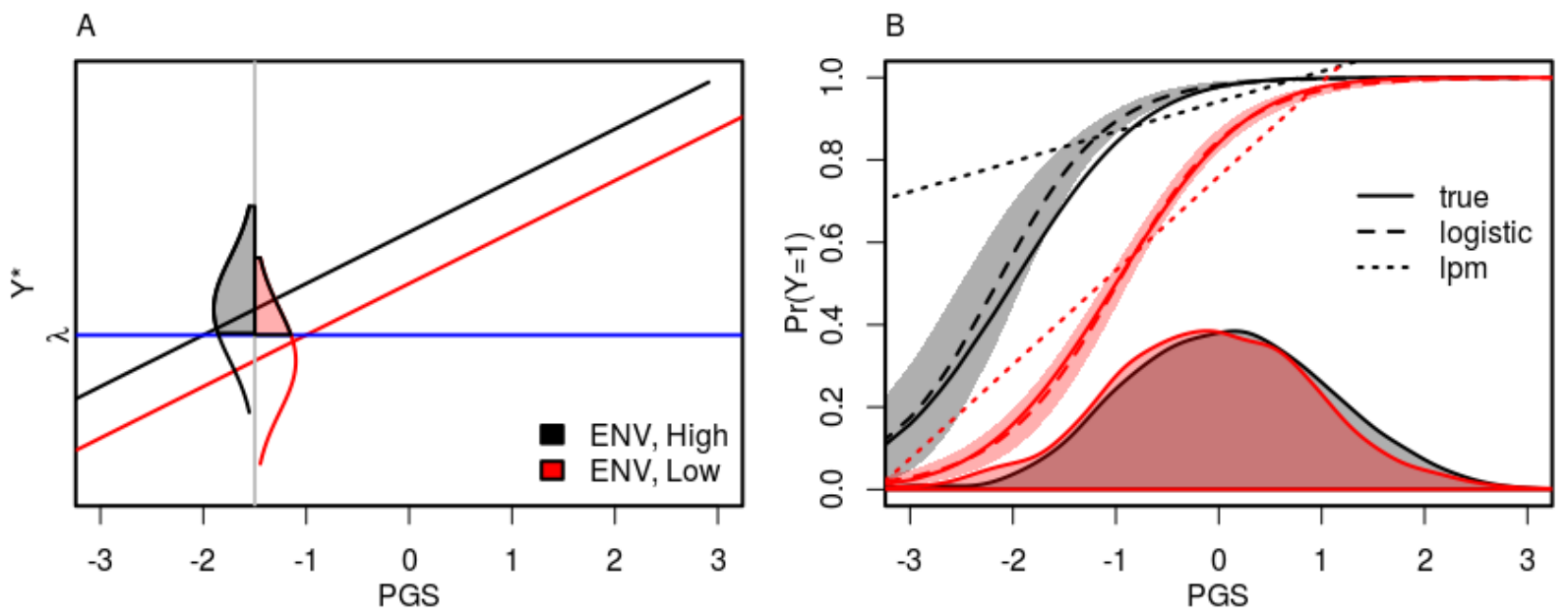

\section{C. Measurement error \& statistical power}

Measurement error acts to both bias associated parameter estimates towards zero (50) and to distort power calculations. In the specific context of GxE studies, there are several concerns. Measurement error exists in both the operationalized PGS and ENV variables of Eqn 2. Measurement error in $\mathrm{G}$, which results from imprecise estimates of the GWAS betas used to construct the PGS, has received some attention (51-53). However, less attention has been paid to measurement error in E. Homoscedastic measurement error in E has substantial implications for power (matters may be further complicated in the presence of non-homoscedastic measurement error, but we focus on the simpler case here). Figure 2 is a simple illustration of this via a simulation study. ${ }^{5}$ We assume that we measure both the PGS and the target environmental variable with error. We focus on variation in the reliability of the environmental measure (the $\mathrm{x}$ axis) and choose two levels of reliability (which we index as alpha) of 0.25 (on left) and 0.5 (on right) for the PGS; we view these reliabilities as representative of relatively weak and relatively strong polygenic scores given existing GWAS. The main takeaway is that ignoring measurement error with respect to the environment inevitably leads to inflated power calculations.

\footnotetext{
${ }^{5}$ Code available here
} 
Let us first focus our attention on a PGS with relatively high reliability by current PGS standards (alpha $=0.5)$ in the case where we have 1000 respondents. We first assume that there is no error in our environmental measure (region emphasized in gray rectangle). In such a case, power is below standard levels of acceptability (power=0.8). As the reliability of our environmental measure declines, however, power becomes increasingly poor. Even when the environment is measured with decent reliability (alpha=0.7), power is greatly reduced (power 0.4). In the case where the PGS is of lower reliability, power is even worse (power 0.2 for an environmental measure of reliability alpha=0.7). When the PGS is measured with substantial error (alpha=0.25), even relatively large samples (when considering population-based studies) of $\mathrm{N}=10,000$ will suffer from power limitations when the environment is also measured with substantial error. These calculations are based upon a toy model that might not be relevant in all cases, but given that interaction studies are power-hungry even without considering measurement error (54), our model emphasizes the need to carefully consider whether one has reasonable power before conducting GxE studies.

Figure 2. Power reduction as a function of measurement error in both PGS and ENV. Left and right panels focus on relatively low $(\mathrm{alpha}=0.25)$ and high $(\mathrm{alpha}=0.5$ ) reliability polygenic scores.
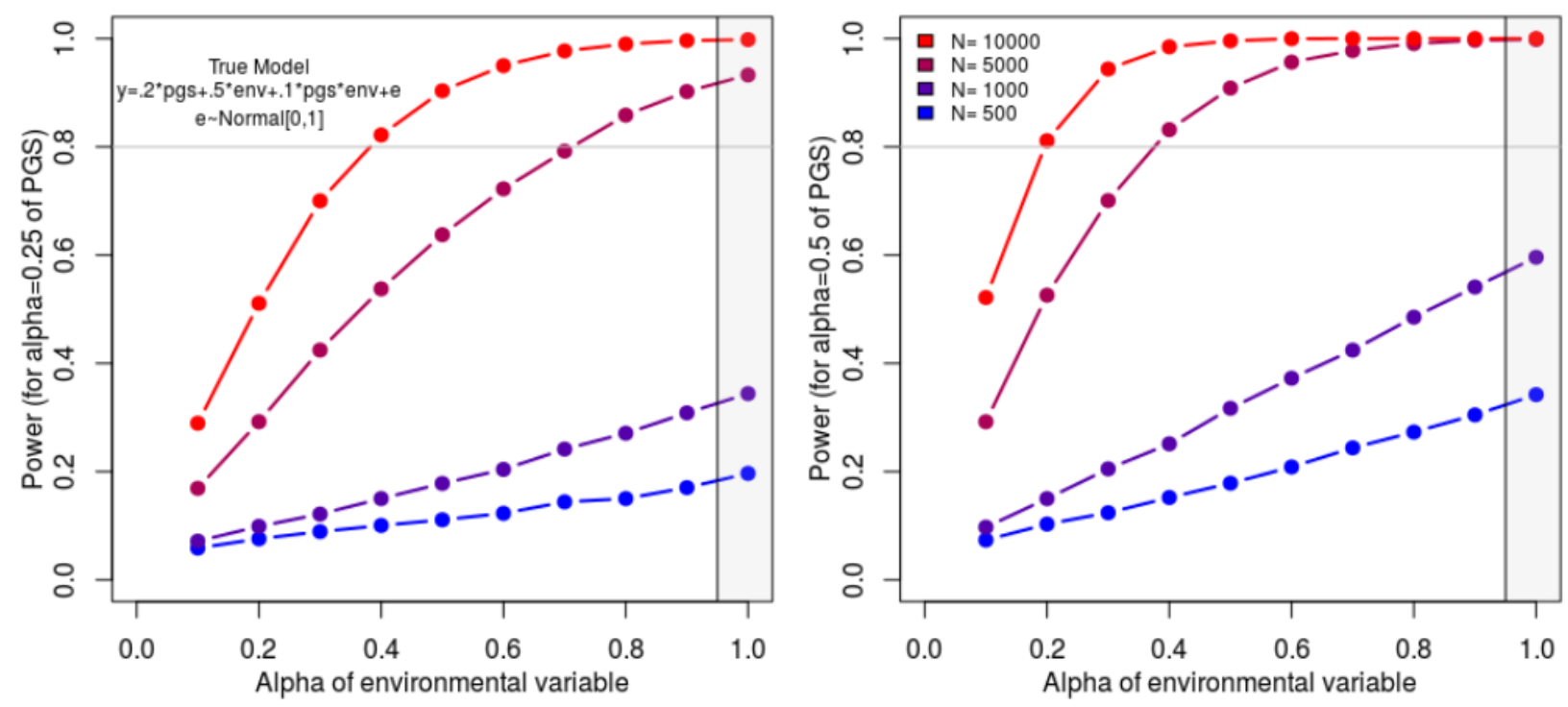

\section{D. Sample selection processes and internal and external validity}


Selection processes complicate inference in observational settings in a number of ways, and studies of GxE are no exception. An often-underappreciated point is that sample selection issues threaten both external and internal validity. We discuss several (potentially overlapping) types of selection that are particularly relevant for GxE research. These sample selection processes limit the population to which GxE findings can be generalized and may lead to spurious results via collider bias (55). Notably, sample selection may pose a threat both in the discovery GWAS used to identify the betas needed to construct a PGS and in the prediction sample in which the PGS is actually constructed and used to estimate GxE.

We begin with mortality selection. Such selection occurs when a non-random subset is lost to mortality and therefore not observed. In studies of older respondents (e.g., the HRS), mortality selection tends to makes the resulting sample "healthier, wealthier, and wiser" (56). Mortality selection is especially relevant to GxE research because genotyping is a relatively recent technology; participants in longstanding cohort studies needed to survive long enough to make it into the genotyped subsample. Indeed, there is evidence to suggest that GxE findings may be sensitive to the presence of mortality selection (57). When studying health-related traits, especially in older populations, we need to consider mortality selection's role in shaping findings (58). In scenarios wherein mortality can be readily modeled with existing data, one possible analytic solution is to use inverse probability weighting (59) to correct for the role of mortality selection. A related issue is that individuals with certain genetic profiles-e.g., those with high genetic liabilities for schizophrenia - may be under-represented in various data sources $(26,60$ 62). Such selection can also lead to issues of both bias and generalizability in subsequent studies. A second issue is that demographic factors play a role in who participates in genetic studies. This, in turn, has implications with respect to the populations to which results using genetic subsamples may generalize. Of particular note is the massive overrepresentation of European-descent individuals in both GWAS (63) and PGS (64) studies. This problem is due to several factors, including both the overrepresentation of European-descent individuals in genetic studies and the fact that differences in linkage disequilibrium across groups leads to the GWAS findings performing better in the (predominantly European) samples from which they are derived. Efforts (65) are underway to monitor (with the hope of then remedying) this problem. In the meantime, researchers have noted that adoption of polygenic scores in precision medicine 
may exacerbate pre-existing health disparities (66). Equity concerns need to be in the foreground of genetics research; GxE is no exception.

These selection problems offer both internal and external validity threats to GxE studies that researchers should carefully consider. An additional concern is that non-random selection into the analytic samples used in empirical studies may lead to reduced environmental variation, further challenging attempts to make accurate inferences regarding GxE. As an illustration, we consider two key adolescent environments - the socioeconomic circumstances of home (67) and the disadvantage of one's residential neighborhood (68), both from Wave I of Add Health (69) that may be of interest. As a function of the way the analytic sample becomes a selected portion of the full sample, we observe a decrease in environmental variance. These decreases will lead to even further reductions in our power to detect GxE effects; in particular, power analyses motivated by environmental variation observed in the full sample are likely to overstate true power given that empirical work will then take place with reduced environmental variation. Beyond power concerns, such selection can lead to a reduction in density in certain regions of the distribution of the measured environment that will increase the challenge of identifying the relevant functional form in that region.

Figure 3. Distributions of two key environmental variables (household SES and neighborhood disadvantage) taken from Wave I of Add Health (69). Note the reduction in variation of the distribution for the analytic sample (in red) versus that of the full sample (in blue). Reductions in the standard deviation are $11 \%$ for SES and 14\% for neighborhood disadvantage; in variance terms, the reductions are $20 \%$ and $26 \%$ respectively.
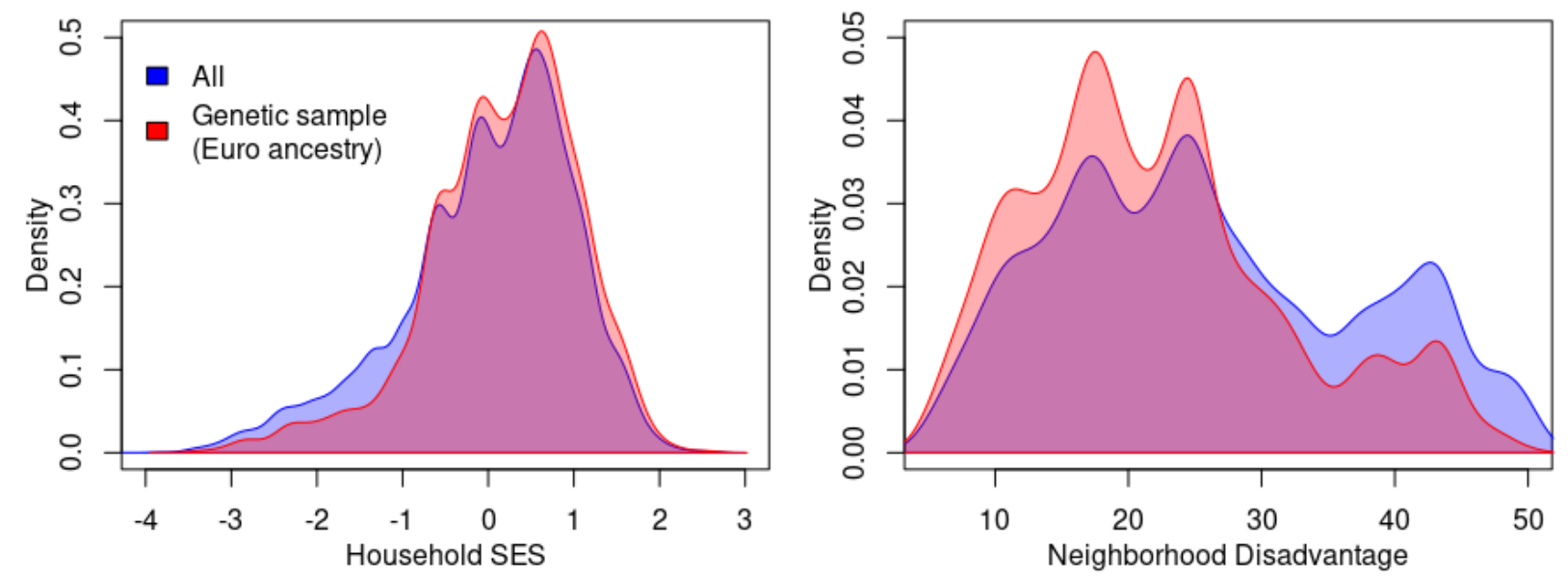


\section{Suggestions for improving design of GxE studies}

Below we discuss several considerations that researchers conducting GxE work may find valuable. We acknowledge at the outset that these are a relatively modest set of recommendations. However, we think they are still important. From both a conceptual understanding of GWAS design and its implications for polygenic score performance as well as our on-the-ground experience, we anticipate that polygenic scores will tend to be fairly invariant predictors unless environments that have strong main effects on the outcome are being considered. Our suggestions stem from this perspective.

Power analyses should be the norm (and not the exception) in GxE research. Traditional power analyses are used to inform key design features, such as the sample size, prior to the implementation of a study. In contrast, power analyses of the type considered here (i.e., posthoc) offer information about the power of a study design given existing data (e.g., the sample sizes available from large cohort studies such as the HRS and Add Health) and key assumptions about the relevant parameters. Such analyses are not cure-alls but will hopefully make researchers more attentive to the limitations that they face (specifically, the likelihood of observing false positives) in a given context.

We also emphasize the potential for analyses that take advantage of environmental variation without specifying a specific environmental feature of interest. In situations wherein individuals cluster into some unit, researchers may first want to consider the level of empirical support for GxE based on relatively omnibus measures of the environment. For example, one might test for variation observed in the relationship between phenotype and polygenic score across environmental units (e.g. schools or census tracts); see (49) for one such example. Such analyses are informative in that they offer preliminary guidance on whether specific features of the environments deserve additional scrutiny as possible GxE targets. Yet another approach that researchers might want to consider leverages changing patterns of heritability (70).

Finally, researchers should be more attentive to the issue of what form of GxE to expect; e.g. does the candidate environment operate as a lens, a dimmer, or take on some more complex functional form? GxE research will, in our experience, tend to observe that environments with 
large main effects on a phenotype act as dimmers. In other words, they will moderate with the magnitude of the effect of the polygenic score on the outcome, without changing its sign. Such observations may be of high value, but they are also potentially quite distinct from more dramatic patterns of sign reversal of PGS effects in different environments that have received a great deal of conceptual attention. In studies seeking to identify lens-type patterns (71), both the genetic and the environmental measures are of crucial importance for testing such hypotheses in which the environmental context determines whether a given genotype is risky or advantageous .

\section{Conclusion}

GxE characterizes both the environmental contingency of genetically-linked processes and the genetic contingency of environmentally-linked processes. In our view, GxE studies involving human behavior and polygenic scores may offer valuable insights but are also at-risk of repeating many of the mistakes made by previous eras of research (e.g., the candidate gene era). Our goal has been to emphasize the need for careful thinking about the rationale and methods underlying investigations of GxE.

The issues raised here are not an exhaustive enumeration of the challenges of GxE research. However, careful attention to the issues highlighted is likely to produce higher quality research. An overarching goal of research examining the combined genetic and environmental contributions to human behavior is to help construct useful models of human behavior. In our view, useful models avoid unnecessary complexity when accounting for messy data. At its best, GxE research can help inform the construction of such models by parsimoniously showcasing complexities from empirical reality that need to be accounted for. For instance, GxE research can help reveal important heterogeneity in developmental processes, treatment responses, and policy effects. To be informative, however, we must exercise care. Otherwise, GxE research threatens to introduce confusion into the already challenging problem of understanding human behavior. 


\section{References}

1. Visscher PM, Wray NR, Zhang Q, Sklar P, McCarthy MI, Brown MA, et al. 10 Years of GWAS Discovery: Biology, Function, and Translation. Am J Hum Genet. 2017 Jul 6;101(1):5-22.

2. Pearson TA, Manolio TA. How to interpret a genome-wide association study. Jama. 2008;299(11):1335-1344.

3. Boyle EA, Li YI, Pritchard JK. An Expanded View of Complex Traits: From Polygenic to Omnigenic. Cell. 2017;169(7):1177-1186.

4. Chabris CF, Lee JJ, Cesarini D, Benjamin DJ, Laibson DI. The Fourth Law of Behavior Genetics. Curr Dir Psychol Sci. 2015 Aug 1;24(4):304-12.

5. Dudbridge F. Power and predictive accuracy of polygenic risk scores. PLoS Genet. 2013;9(3):e1003348.

6. Dudbridge F. Polygenic Epidemiology. Genet Epidemiol. 2016 May 1;40(4):268-72.

7. Cesarini D, Visscher PM. Genetics and educational attainment. Npj Sci Learn [Internet]. 2017 Dec [cited 2018 Sep 25];2(1). Available from: http://www.nature.com/articles/s41539-017-0005-6

8. Lee JJ, Wedow R, Okbay A, Kong E, Maghzian O, Zacher M, et al. Gene discovery and polygenic prediction from a genome-wide association study of educational attainment in 1.1 million individuals. Nat Genet. 2018 Aug;50(8):1112-21.

9. Sugrue LP, Desikan RS. What are polygenic scores and why are they important? Jama. 2019;321(18):1820-1821.

10. Harden KP, Koellinger PD. Using genetics for social science. Nat Hum Behav. 2020;1-10.

11. Belsky DW, Harden KP. Phenotypic Annotation: Using Polygenic Scores to Translate Discoveries From Genome-Wide Association Studies From the Top Down. Curr Dir Psychol Sci. 2019 Jan 9;096372141880772.

12. Conley D, Fletcher J. The Genome Factor: What the Social Genomics Revolution Reveals about Ourselves, Our History, and the Future. Princeton: Princeton University Press; 2017.

13. Mills MC, Tropf FC. Sociology, Genetics, and the Coming of Age of Sociogenomics. Annu Rev Sociol. 2020 Jul 30;46(1):annurev-soc-121919-054756.

14. Feldman MW, Lewontin RC. The heritability hang-up. Science. 1975 Dec 19;190(4220):1163-8.

15. Purcell S. Variance components models for gene-environment interaction in twin analysis. Twin Res Hum Genet. 2002;5(6):554-571. 
16. Duncan LE, Keller MC. A critical review of the first 10 years of candidate gene-byenvironment interaction research in psychiatry. Am J Psychiatry. 2011 Oct;168(10):1041-9.

17. Hewitt JK. Editorial policy on candidate gene association and candidate gene-byenvironment interaction studies of complex traits. Behav Genet. 2012;42(1):1.

18. Ware EB, Schmitz LL, Faul JD, Gard A, Mitchell C, Smith JA, et al. Heterogeneity in polygenic scores for common human traits. bioRxiv. 2017 Feb 5;106062.

19. Braudt DB, Harris KM. Polygenic scores (PGSs) in the national longitudinal study of adolescent to adult health (Add Health)-Release 1. Carol Digit Repos. 2018;1-72.

20. Okbay A, Benjamin D, Visscher P. Documentation (Construction of WLS Polygenic Scores) [Internet]. 2018. Available from:

https://www.ssc.wisc.edu/wlsresearch/documentation/GWAS/Lee_et_al_(2018)_PGS_WL S.pdf

21. Lambert SA, Gil L, Jupp S, Ritchie SC, Xu Y, Buniello A, et al. The Polygenic Score Catalog: an open database for reproducibility and systematic evaluation [Internet]. Genetic and Genomic Medicine; 2020 May [cited 2020 May 26]. Available from:

http://medrxiv.org/lookup/doi/10.1101/2020.05.20.20108217

22. Polderman T, Benyamin B, De Leeuw CA, Sullivan PF, Van Bochoven A, Visscher PM, et al. Meta-analysis of the heritability of human traits based on fifty years of twin studies. Nat Genet. 2015;47(7):702-709.

23. Rosenquist JN, Lehrer SF, O’Malley AJ, Zaslavsky AM, Smoller JW, Christakis NA. Cohort of birth modifies the association between FTO genotype and BMI. Proc Natl Acad Sci. 2015;112(2):354-359.

24. Boardman JD, Barnes LL, Wilson RS, Evans DA, de Leon CFM. Social disorder, APOEE4 genotype, and change in cognitive function among older adults living in Chicago. Soc Sci Med. 2012;74(10):1584-1590.

25. Mostafavi H, Harpak A, Agarwal I, Conley D, Pritchard JK, Przeworski M. Variable prediction accuracy of polygenic scores within an ancestry group. Elife. 2020;9.

26. Pirastu N, Cordioli M, Nandakumar P, Mignogna G, Abdellaoui A, Hollis B, et al. Genetic analyses identify widespread sex-differential participation bias. BioRxiv. 2020;

27. Morris TT, Davies NM, Hemani G, Smith GD. Population phenomena inflate genetic associations of complex social traits. Sci Adv. 2020;6(16):eaay0328.

28. Boardman JD, Daw J, Freese J. Defining the environment in gene-environment research: lessons from social epidemiology. Am J Public Health. 2013;103(S1):S64-S72.

29. Jaffee SR, Price TS. Gene-environment correlations: a review of the evidence and implications for prevention of mental illness. Mol Psychiatry. 2007;12(5):432-442. 
30. Briley DA, Livengood J, Derringer J, Tucker-Drob EM, Fraley RC, Roberts BW. Interpreting Behavior Genetic Models: Seven Developmental Processes to Understand. Behav Genet. 2019 Mar;49(2):196-210.

31. Dudbridge F, Fletcher O. Gene-Environment Dependence Creates Spurious GeneEnvironment Interaction. Am J Hum Genet. 2014 Sep;95(3):301-7.

32. Fletcher JM, Conley D. The challenge of causal inference in gene-environment interaction research: Leveraging research designs from the social sciences. Am J Public Health. 2013;103(S1):S42-S45.

33. Schmitz L, Conley D. The Long-Term Consequences of Vietnam-Era Conscription and Genotype on Smoking Behavior and Health. Behav Genet. 2016;46(1):43-58.

34. Barcellos SH, Carvalho LS, Turley P. Education can reduce health differences related to genetic risk of obesity. Proc Natl Acad Sci. 2018 Oct 16;115(42):E9765-72.

35. Schmitz LL, Conley D. The effect of Vietnam-era conscription and genetic potential for educational attainment on schooling outcomes. Econ Educ Rev. 2017;61:85-97.

36. Khera AV, Chaffin M, Wade KH, Zahid S, Brancale J, Xia R, et al. Polygenic Prediction of Weight and Obesity Trajectories from Birth to Adulthood. Cell. 2019 Apr;177(3):587596.e9.

37. Lubinski D, Humphreys LG. Assessing spurious" moderator effects": Illustrated substantively with the hypothesized ("synergistic") relation between spatial and mathematical ability. Psychol Bull. 1990;107(3):385.

38. MacCallum RC, Mar CM. Distinguishing between moderator and quadratic effects in multiple regression. Psychol Bull. 1995;118(3):405.

39. Keller MC. Gene $\times$ Environment Interaction Studies Have Not Properly Controlled for Potential Confounders: The Problem and the (Simple) Solution. Biol Psychiatry. 2014 Jan $1 ; 75(1): 18-24$.

40. Johnson SB, Riis JL, Noble KG. State of the art review: poverty and the developing brain. Pediatrics. 2016;137(4):e20153075.

41. Duncan GJ, Magnuson K. Socioeconomic status and cognitive functioning: moving from correlation to causation. Wiley Interdiscip Rev Cogn Sci. 2012;3(3):377-386.

42. Barr PB, Silberg J, Dick DM, Maes HH. Childhood socioeconomic status and longitudinal patterns of alcohol problems: Variation across etiological pathways in genetic risk. Soc Sci Med. 2018;209:51-58.

43. Musci RJ, Bettencourt AF, Sisto D, Maher B, Masyn K, Ialongo NS. Violence exposure in an urban city: A GxE interaction with aggressive and impulsive behaviors. J Child Psychol Psychiatry. 2019;60(1):72-81. 
44. Gould KL, Coventry WL, Olson RK, Byrne B. Gene-environment interactions in ADHD: the roles of SES and chaos. J Abnorm Child Psychol. 2018;46(2):251-263.

45. Belsky J, Pluess M. Beyond diathesis stress: Differential susceptibility to environmental influences. Psychol Bull. 2009;135(6):885.

46. Ellis BJ, Boyce WT, Belsky J, Bakermans-Kranenburg MJ, Van IJzendoorn MH.

Differential susceptibility to the environment: An evolutionary-neurodevelopmental theory. Dev Psychopathol. 2011;23(01):7-28.

47. Obradović J, Boyce WT. Individual differences in behavioral, physiological, and genetic sensitivities to contexts: Implications for development and adaptation. Dev Neurosci. 2009;31(4):300-308.

48. Widaman KF, Helm JL, Castro-Schilo L, Pluess M, Stallings MC, Belsky J. Distinguishing ordinal and disordinal interactions. Psychol Methods. 2012;17(4):615.

49. Trejo S, Belsky D, Boardman J, Freese J, Harris K, Herd P, et al. Schools as Moderators of Genetic Associations with Life Course Attainments: Evidence from the WLS and Add Health. Sociol Sci. 2018;5:513-40.

50. Hutcheon JA, Chiolero A, Hanley JA. Random measurement error and regression dilution bias. BMJ. 2010 Jun 23;340(jun23 2):c2289-c2289.

51. Conley D, Laidley TM, Boardman JD, Domingue BW. Changing Polygenic Penetrance on Phenotypes in the 20th Century Among Adults in the US Population. Sci Rep. 2016;6:30348.

52. DiPrete TA, Burik CAP, Koellinger PD. Genetic instrumental variable regression: Explaining socioeconomic and health outcomes in nonexperimental data. Proc Natl Acad Sci. 2018 May 29;115(22):E4970-9.

53. Tucker-Drob EM. Measurement Error Correction of Genome-Wide Polygenic Scores in Prediction Samples [Internet]. Genetics; 2017 Jul [cited 2019 Apr 19]. Available from: http://biorxiv.org/lookup/doi/10.1101/165472

54. McClelland GH, Judd CM. Statistical difficulties of detecting interactions and moderator effects. Psychol Bull. 1993;114(2):376-90.

55. Elwert F, Winship C. Endogenous selection bias: The problem of conditioning on a collider variable. Annu Rev Sociol. 2014;40:31-53.

56. Zajacova A, Burgard SA. Healthier, wealthier, and wiser: a demonstration of compositional changes in aging cohorts due to selective mortality. Popul Res Policy Rev. 2013;32(3):311324. 
57. Domingue BW, Belsky DW, Harrati A, Conley D, Weir D, Boardman J. Mortality Selection in a Genetic Sample and Implications for Association Studies. Int J Epidemiol. 2017;46:1285-94.

58. Oliynyk RT. Age-related late-onset disease heritability patterns and implications for genome-wide association studies. PeerJ. 2019;7:e7168.

59. van der Wal WM, Geskus RB. IPW: an R package for inverse probability weighting. J Stat Softw. 2011;43(13):1-23.

60. Martin J, Tilling K, Hubbard L, Stergiakouli E, Thapar A, Davey Smith G, et al. Association of Genetic Risk for Schizophrenia With Nonparticipation Over Time in a Population-Based Cohort Study. Am J Epidemiol. 2016 Jun 15;183(12):1149-58.

61. Meisner A, Kundu P, Chatterjee N. Case-only analysis of gene-environment interactions using polygenic risk scores [Internet]. Genetics; 2019 Feb [cited 2019 Apr 19]. Available from: http://biorxiv.org/lookup/doi/10.1101/555300

62. Taylor AE, Jones HJ, Sallis H, Euesden J, Stergiakouli E, Davies NM, et al. Exploring the association of genetic factors with participation in the Avon Longitudinal Study of Parents and Children. Int J Epidemiol. 2018 Aug 1;47(4):1207-16.

63. Mills MC, Rahal C. A scientometric review of genome-wide association studies. Commun Biol. 2019 Dec;2(1):9.

64. Duncan L, Shen H, Gelaye B, Meijsen J, Ressler K, Feldman M, et al. Analysis of polygenic risk score usage and performance in diverse human populations. Nat Commun. 2019;10(1):3328.

65. Mills MC, Rahal C. The GWAS Diversity Monitor tracks diversity by disease in real time. Nat Genet. 2020;52(3):242-243.

66. Martin AR, Kanai M, Kamatani Y, Okada Y, Neale BM, Daly MJ. Clinical use of current polygenic risk scores may exacerbate health disparities. Nat Genet. 2019;51(4):584.

67. Belsky D, Domingue B, Wedow R, Arseneault L, Boardman J, Caspi A, et al. Genetic analysis of social-class mobility in five longitudinal studies. Proc Natl Acad Sci. 2018;

68. Belsky DW, Caspi A, Arseneault L, Corcoran DL, Domingue BW, Harris KM, et al. Genetics and the geography of health, behaviour and attainment. Nat Hum Behav [Internet]. 2019 Apr 8 [cited 2019 Apr 22]; Available from: http://www.nature.com/articles/s41562019-0562-1

69. Harris KM, Halpern CT, Whitsel EA, Hussey JM, Killeya-Jones LA, Tabor J, et al. Cohort Profile: The National Longitudinal Study of Adolescent to Adult Health (Add Health). Int J Epidemiol. 2019 Jun 29; dyz115. 
70. Tropf FC, Lee SH, Verweij RM, Stulp G, Most PJ van der, Vlaming R de, et al. Hidden heritability due to heterogeneity across seven populations. Nat Hum Behav. 2017 Sep 11;1.

71. Troth A, Puzey JR, Kim RS, Willis JH, Kelly JK. Selective trade-offs maintain alleles underpinning complex trait variation in plants. Science. 2018;361(6401):475-478. 